\section{Absenteísmo por doença ocupacional de trabalhadores rurais no setor canavieiro}

\author{
Absenteeism due to occupational diseases among \\ sugarcane workers
}

\author{
Absentismo laboral por enfermedad profesional de \\ los trabajadores rurales en la industria de la caña \\ de azúcar
}

Aline Duarte Ferreira Ceccato 1

Luiz Carlos Soares de Carvalho Junior 1 Rafaela Campos Cuissi 1

Mariane Monteschi 1

Nayara Galvão Oliveira 1

Carlos Roberto Padovani 1

Ercy Mara Cipulo Ramos 1

Dionei Ramos 1

\footnotetext{
${ }^{1}$ Faculdade de Ciências e Tecnologia, Universidade Estadual Paulista Júlio de Mesquita Filho, Presidente Prudente, Brasil.

Correspondência

A. D. F. Ceccato

Faculdade de Ciências e

Tecnologia, Universidade Estadual Paulista Júlio de Mesquita Filho.

Rua Roberto Simonsen 305 Presidente Prudente, SP 19060-900, Brasil.

alineduarteferreira@hotmail. com
}

\begin{abstract}
The aim of this study was to analyze the frequency of work-related sick leave in sugarcane workers. A total of 1,230 medical excuses for 400 sugarcane workers were analyzed according to the International Classification of Diseases, 10th Revision (ICD-10). The following items were analyzed: diagnosis, sex, and length and season of sick leave. In all the seasons, musculoskeleta diseases showed the highest sick leave rate, following by respiratory diseases $(p<0.05)$. Sick leave due to musculoskeletal diseases was more frequent at the end of the sugarcane harvest than during the intercrop season ( $p<0.05)$. Seventy-five percent of medical excuses were for one day. The longest sick leaves were for diseases of the genitourinary tract $(p<0.001)$. It is thus essential to have a multidisciplinary health team focused on the development of ergonomic activities, personal protective equipment, and health orientation specifically targeting this group's needs.
\end{abstract}

Rural Workers; Absenteeism; Occupational Diseases

\section{Resumo}

O objetivo do estudo foi analisar a frequência de afastamentos por doenças ocupacionais de trabalhadores rurais em uma safra canavieira. Foram analisados 1.230 atestados médicos classificados de acordo com a 10a revisão da Classificação Internacional de Doenças (CID-10) de 400 trabalhadores rurais. Nos atestados foram analisados: diagnóstico médico, sexo, dias de afastamento $e$ período do afastamento. Em todos os períodos as doenças osteomusculares apresentaram maior frequência de atestados seguidos das doenças respiratórias $(p<0,05)$. Os atestados por doenças osteomusculares foram mais frequentes no fim da safra do que na entressafra $(p<0,05)$. Em relação ao número de dias afastados, $75 \%$ dos atestados foram de um dia. Os atestados por doenças do aparelho geniturinário obtiveram maior número de dias afastados $(p<0,001)$. Assim, é fundamental a atuação de uma equipe multidisciplinar de profissionais da saúde que atentem para o desenvolvimento de atividades de ergonomia, equipamentos de proteção individual e orientações de saúde direcionadas especificamente às necessidades dessa população.

Trabalhadores Rurais; Absenteísmo; Doenças Profissionais 


\section{Introdução}

O setor de produção de açúcar e álcool é um dos mais importantes na geração de empregos atualmente no Brasil; estima-se que cerca de 500 mil trabalhadores estão empregados nesse setor 1 .

A produção de biocombustível na indústria sucroalcooleira é dividida em duas fases: fase agrícola e fase industrial 2. A fase agrícola é dividida em dois períodos: entressafra e safra. A entressafra é caracterizada pelo plantio da cana crua e não ocorre a queima da palha da cana-deaçúcar e, nesse período, os trabalhadores são expostos à grande quantidade de poeira ocupacional, enquanto na safra ocorre a queima noturna da palha da cana-de-açúcar, fato que antecede o corte manual, realizado pelo cortador de cana 3 .

O incentivo à produção de açúcar e álcool advindos da cana-de-açúcar tem estimulado a geração em ritmo acelerado de novas oportunidades de trabalho, com crescente demanda por trabalhadores rurais. Tais mudanças ocorridas na dinâmica do trabalho nas indústrias de açúcar e álcool podem agravar os impactos relacionados à saúde dos trabalhadores rurais envolvidos 4 .

A saúde dos trabalhadores rurais que atuam no setor canavieiro sofre influência das condições ambientais a que são expostos, e a abordagem dessa temática permite analisar a gênese de uma série de agravantes da integridade de sua saúde havendo riscos de ordem física, química, biológica, ergonômica, mecânica e psicológica 5 . Os agravos ocupacionais que atingem essa parcela da população decorrem, em grande parte, do esforço físico excessivo ${ }^{6}$. Além disso, acidentes com máquinas e animais peçonhentos, envenenamentos por agrotóxicos e frequência de doenças respiratórias por exposição aos poluentes gerados pela queima da palha da cana-de-açúcar são fatores que contribuem para o absenteísmo 5 .

Atualmente, a influência do trabalho como fator causal de dano ou agravo à saúde está bem estabelecida e dimensionada em sua importância e magnitude. O absenteísmo no ambiente de trabalho rural causado por doenças ocupacionais reflete o estado de saúde dos trabalhadores, além de causar impactos econômicos importantes no setor e gerar custos elevados às empresas e à seguridade social. No entanto, a frequência de afastamentos e as suas principais causas são pouco discutidas, especialmente nos países em desenvolvimento, o que dificulta a elaboração de programas de prevenção e reabilitação voltados para essa população 7,8 .

O estudo dos afastamentos do trabalho por doença ocupacional é imprescindível para fundamentar discussões sobre a condição de saúde e doença dos trabalhadores, assim como elabo- rar políticas de promoção, prevenção e reabilitação da saúde 4 . No Brasil, levantamentos sobre o perfil de morbimortalidade de trabalhadores rurais da indústria canavieira são insuficientes para conhecer adequadamente as características dessa população em relação ao processo saúdedoença-trabalho 8,9. Dentro desse contexto, o estudo analisou a frequência de afastamentos por doenças ocupacionais de trabalhadores rurais ao longo de uma safra canavieira.

\section{Métodos}

Estudo transversal retrospectivo predominantemente descritivo realizado com trabalhadores rurais registrados em uma usina sucroalcooleira localizada na região oeste do Estado de São Paulo, Brasil. O banco de dados do presente estudo foi elaborado com base nos arquivos de atestados de saúde ocupacional (ASO) referentes ao ano de 2010.

Foram observadas as seguintes variáveis: sexo; diagnóstico médico das doenças ocupacionais; função de trabalho; dias de afastamento e período do afastamento (entressafra, meio de safra e fim de safra). O diagnóstico médico das doenças que originaram os afastamentos foi codificado segundo a 10a revisão da Classificação Internacional de Doenças (CID-10) 10.

Foi analisado um total de 1.230 atestados médicos de 400 trabalhadores rurais (343 homens e 57 mulheres), entre os meses de fevereiro e outubro de 2010. Esses trabalhadores rurais exerciam funções variadas, entre elas a de plantio e corte manual da cana-de-açúcar (entressafra e safra respectivamente), controle de pesticidas e queima noturna da cana-de-açúcar (período de safra canavieira).

Os atestados médicos foram organizados e separados de acordo com os períodos de entressafra, meio e final de safra. A entressafra, caracterizada pelo período de plantio da cana crua sem a queima, foi delimitada entre os meses de fevereiro a abril. A safra, caracterizada pela colheita manual da cana queimada, foi separada em dois períodos: meio da safra (correspondente aos meses de maio e julho) e final da safra (correspondente aos meses de agosto a outubro).

As doenças ocupacionais foram divididas em nove grupos e classificadas de acordo com a CID10: doenças do sistema osteomuscular e do tecido conjuntivo; doenças do aparelho respiratório; doenças do sistema nervoso; outros sintomas e sinais gerais; dor não especificada; doenças da pele e do tecido subcutâneo; doenças do aparelho geniturinário; outros transtornos dos dentes e de suas estruturas de sustentação; lesões e en- 
venenamentos e outras consequências de causas externas. Neste estudo foram excluídos os afastamentos que não apresentaram relação entre a causa do afastamento e a atividade exercida no setor canavieiro.

A análise dos afastamentos levou em consideração as variáveis referentes ao número de dias afastados, o período de afastamento e a causa do absenteísmo ocupacional. O estudo contou com a aprovação do Comitê de Ética em Pesquisa da Faculdade de Ciência e Tecnologia, Universidade Estadual Paulista (parecer no 15/2010).

Para a análise estatística, foi utilizado o SPSS, versão 16.0 para Windows (SPSS Inc., Chicago, Estados Unidos). Para a análise das medidas descritivas dos dias afastados, segundo o período e a CID-10, utilizou-se o teste não paramétrico de Kruskal-Wallis, seguido do pós-teste de Dunn. Para a análise do número de dias de afastamento, segundo o gênero, utilizou-se o teste não paramétrico de Mann-Whitney. O teste de Goodman 11 foi utilizado para identificar a frequência de trabalhadores que apresentaram afastamentos médicos em cada classe de doença nos períodos, assim como para determinar a frequência dos atestados de cada classe de doença apresentados por cada gênero e a frequência de afastamentos de cada gênero nos três períodos estudados. O nível de significância considerado foi de $5 \%(p<0,05)$.

\section{Resultados}

O estudo analisou um total de 1.230 atestados médicos apresentados por 400 trabalhadores rurais do setor canavieiro ao longo de um ano. A análise levou em consideração o tipo de doença ocupacional, os períodos de afastamento e o número de dias afastados.

A Tabela 1 representa a frequência do número de atestados entre as doenças ocupacionais relacionadas ao período. Na entressafra, a frequência de atestados foi maior entre as doenças do sistema osteomuscular e do tecido conjuntivo, seguidas das doenças do aparelho respiratório $(p<0,05)$. No meio da safra, houve maior frequência no número de atestados de doenças do sistema osteomuscular e do tecido conjuntivo; em segundo lugar estão as doenças do aparelho respiratório; e, em terceiro lugar, as doenças do sistema nervoso $(p<0,05)$. No fim da safra. a frequência no número de atestados foi maior nas doenças do sistema osteomuscular e do tecido conjuntivo e, em segundo lugar, as doenças do aparelho respiratório $(\mathrm{p}<0,05)$.

Na comparação entre a entressafra com o fim da safra, constatou-se que houve aumento sig- nificante na frequência dos atestados no fim da safra para o grupo das doenças do sistema osteomuscular e do tecido conjuntivo. A Tabela 2 representa a distribuição do número de atestados ocupacionais segundo o gênero, somados todos os períodos. Ocorreu diferença significante entre os gêneros nas doenças do aparelho geniturinário, no qual o sexo feminino apresentou maior número de atestados em relação ao sexo masculino $(\mathrm{p}<0,05)$. No grupo de doenças referentes a outros sintomas e sinais relativos às sensações e às percepções gerais, o sexo masculino apresentou maior número de atestados em relação ao sexo feminino $(\mathrm{p}<0,05)$. No sexo masculino, as doenças que mais apresentaram número de atestados foram as do sistema osteomuscular e do tecido conjuntivo; em segundo lugar, as doenças do aparelho respiratório; e, em terceiro lugar, as doenças do sistema nervoso, as lesões, envenenamentos e outras consequências de causas externas. No sexo feminino, o grupo de doenças que mais apresentou número de atestados foi o das doenças do sistema osteomuscular e do tecido conjuntivo; em segundo lugar, as doenças do aparelho respiratório.

A Tabela 3 representa a relação entre gênero e período de afastamento. Trabalhadores rurais do sexo masculino se afastaram mais nos períodos de meio da safra e fim da safra em relação à entressafra $(p<0,05)$. Nos trabalhadores do sexo feminino, o resultado foi semelhante, ou seja, afastaram-se mais no meio e fim da safra em relação à entressafra $(p<0,05)$. Na comparação entre os gêneros, não houve diferença significante entre os períodos.

Em relação ao número de dias afastados, $75 \%$ dos atestados apresentados pelos trabalhadores foram de um dia de afastamento. As doenças do sistema osteomuscular e do tecido conjuntivo obtiveram 1,86 $\pm 2,25$ dias afastados; o grupo referente às doenças da pele e do tecido subcu-

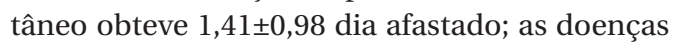
do sistema nervoso tiveram $1,19 \pm 0,50$ dia afastado; os afastamentos que tiveram como causa outros sintomas e sinais gerais tiveram $1,10 \pm 0,30$; as doenças do aparelho respiratório obtiveram $1,25 \pm 0,80$ dia afastado; outros transtornos dos dentes e de suas estruturas de sustentação não classificadas em outra parte $1,16 \pm 0,62$ dia afastado; e as lesões, envenenamentos e outras consequências de causas externas com 2,23 $\pm 2,89$ dias. Os afastamentos referentes às doenças do aparelho geniturinário obtiveram maior número, com $2,71 \pm 3,9(\mathrm{p}<0,001)$. 
Tabela 1

Frequência de atestados apresentados pelos trabalhadores rurais segundo os períodos. Oeste do Estado de São Paulo, Brasil, 2010.

\begin{tabular}{|c|c|c|c|c|c|c|}
\hline \multirow[t]{2}{*}{ Doenças } & \multicolumn{2}{|c|}{ Entressafra * } & \multicolumn{2}{|c|}{ Meio da safra * } & \multicolumn{2}{|c|}{ Fim da safra * } \\
\hline & $\mathbf{n}$ & $\%$ & $\mathbf{n}$ & $\%$ & $\mathbf{n}$ & $\%$ \\
\hline Doenças do sistema osteomuscular e do tecido conjuntivo & $31 \star \star$ & 29,0 & 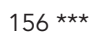 & 30,9 & 254 \#,\#\# & 41,2 \\
\hline Doenças do aparelho respiratório & $23 \# \#$ & 21,5 & $111 \S$ & 22,0 & $110 \S \S$ & 17,9 \\
\hline Doenças do sistema nervoso & 10 & 9,3 & $61 \S \S \S$ & 12,1 & 92 & 14,9 \\
\hline Lesões, envenenamentos e outras consequências de causas externas & 13 & 12,1 & 73 & 14,5 & 57 & 9,3 \\
\hline Dor não especificada & 9 & 8,4 & 39 & 7,7 & 42 & 6,8 \\
\hline Doenças do aparelho geniturinário & 7 & 6,5 & 16 & 3,2 & 15 & 2,4 \\
\hline Outros transtornos dos dentes e de suas estruturas de sustentação & 7 & 6,5 & 23 & 4,6 & 19 & 3,1 \\
\hline Outros sintomas e sinais gerais & 4 & 3,7 & 15 & 3,0 & 12 & 2,0 \\
\hline Doenças da pele e do tecido subcutâneo & 3 & 2,8 & 11 & 2,2 & 15 & 2,4 \\
\hline
\end{tabular}

* A entressafra dura três meses: começa em fevereiro e termina em abril. A safra dura seis meses: começa em abril e termina em outubro. São considerados "meio da safra" os meses de maio a julho. São considerados "fim da safra" os meses de agosto a outubro;

** Maior frequência de atestados na entressafra $(p<0,05)$;

*** Maior frequência de atestados no meio da safra $(p<0,05)$;

\# Maior frequência de atestados no fim da safra $(p<0,05)$;

\#\# Em relação à entressafra $(p<0,05)$;

\#\# Segunda maior frequência de atestados na entressafra $(p<0,05)$;

$\S$ Segunda maior frequência de atestados no meio da safra $(p<0,05)$;

$\S \S$ Terceira maior frequência de atestados no meio da safra $(p<0,05)$;

$\S \S \S$ Segunda maior frequência de atestados no fim da safra $(p<0,05)$.

Tabela 2

Frequência de atestados apresentados pelos trabalhadores rurais segundo o gênero. Oeste do Estado de São Paulo, Brasil, 2010.

\begin{tabular}{|c|c|c|c|c|}
\hline \multirow[t]{2}{*}{ Doenças } & \multicolumn{2}{|c|}{ Masculino } & \multicolumn{2}{|c|}{ Feminino } \\
\hline & $\mathbf{n}$ & $\%$ & $\mathbf{n}$ & $\%$ \\
\hline Doenças do sistema osteomuscular e do tecido conjuntivo & 394 * & 36,9 & 47 ** & 29,4 \\
\hline Doenças do aparelho respiratório & $209 * \star \star$ & 19,6 & $35 \#$ & 21,9 \\
\hline Doenças do sistema nervoso & 145 \#\# & 13,6 & 18 & 11,3 \\
\hline Lesões, envenenamentos e outras consequências de causas externas. & $134 \#$ & 12,6 & 9 & 5,6 \\
\hline Dor não especificada & 75 & 7,0 & 15 & 9,4 \\
\hline Doenças do aparelho geniturinário & 21 & 2,0 & $17 \# \#$ & 10,6 \\
\hline Outros transtornos dos dentes e de suas estruturas de sustentação & 43 & 4,0 & 6 & 3,8 \\
\hline Outros sintomas e sinais gerais & $28 \S$ & 2,6 & 3 & 1,9 \\
\hline Doenças da pele e do tecido subcutâneo & 19 & 1,8 & 10 & 6,3 \\
\hline
\end{tabular}

* Maior frequência de atestados entre o sexo masculino $(p<0,05)$;

** Maior frequência de atestados entre o sexo feminino $(p<0,05)$;

$\star \star \star$ Segunda maior frequência de atestados entre o sexo masculino $(p<0,05)$;

\# Segunda maior frequência de atestados entre o sexo feminino $(p<0,05)$;

\#\# Terceira maior frequência de atestados entre o sexo masculino $(p<0,05)$;

\#\#\# Maior número de atestados em relação ao sexo masculino $(p<0,05)$;

$\S$ Maior número de atestados em relação ao sexo feminino $(p<0,05)$. 
Tabela 3

Frequência de atestados apresentados pelos trabalhadores rurais segundo o gênero nos períodos. Oeste do Estado de São Paulo, Brasil, 2010.

\begin{tabular}{lcccc}
\hline \multirow{2}{*}{ Períodos } & \multicolumn{2}{c}{ Masculino } & \multicolumn{2}{c}{ Feminino } \\
& $\mathbf{n}$ & $\%$ & $\mathbf{n}$ & $\%$ \\
\hline Entressafra * & 85 & 8,0 & 22 & 13,8 \\
Meio da safra * & 436 ** & 40,8 & $69 * *$ & 43,1 \\
Fim da safra * & $547 * *$ & 51,2 & $69 * *$ & 43,1 \\
\hline
\end{tabular}

* A entressafra dura três meses: começa em fevereiro e termina em abril. A safra dura seis meses: começa em abril e termina em outubro. São considerados "meio da safra" os meses de maio a julho. São considerados "fim da safra" os meses de agosto a outubro;

** Em relação à entressafra $(p<0,05)$

\section{Discussão}

Os afastamentos por doenças do sistema osteomuscular e do tecido conjuntivo foram os mais prevalentes nos trabalhadores rurais. Tais achados podem estar associados a fatores de risco como as condições de trabalho inadequadas, adversidades ambientais, alta demanda energética e repetição do trabalho rural nos canaviais, que podem ser os causadores do absenteísmo nessa atividade laboral 6,12.

Em um estudo realizado por Simões et al. 13 com carpinteiros, foi observada a alta frequência de afastamentos por doenças osteomusculares por causa do manuseio da serra elétrica com os membros superiores. Outros estudos que analisaram as condições de trabalho de trabalhadores rurais associaram a alta incidência de absenteísmo por doenças osteomusculares ao sistema de pagamento por produção, caracterizado por altas cargas de trabalho sem pausas, além de ambiente ocupacional propenso ao desenvolvimento de alterações musculoesqueléticas 6,14,15 Esses afastamentos no trabalho por doenças osteomusculares corroboram com os achados do nosso estudo.

Os afastamentos dos trabalhadores rurais por doenças do aparelho respiratório obtiveram a segunda maior frequência neste estudo, o que pode estar associado às condições climáticas adversas, além da exposição direta dos trabalhadores aos poluentes atmosféricos gerados pelas queimas realizadas nos canaviais, que provocam aparecimento de sinais e sintomas respiratórios durante a safra canavieira 16 .

Nas condições climáticas adversas, como a baixa umidade relativa do ar e altas tempera- turas, há uma dificuldade na dispersão de partículas e poluentes atmosféricos no ar, o que torna o ambiente nocivo para os trabalhadores diretamente expostos, propiciando aumento na incidência de comorbidades respiratórias ${ }^{8}$. Em um estudo realizado no campo de trabalho com cortadores de cana-de-açúcar, mostrou-se que a exposição aguda de quatro horas ao material particulado advindo da queima de biomassa foi responsável pela alteração do mecanismo de defesa respiratório desses trabalhadores 17 .

As doenças do sistema nervoso foram a terceira causa mais incidente de afastamentos nesse estudo. $\mathrm{O}$ achado pode estar relacionado com a exposição às substâncias tóxicas presentes em pesticidas e à poluição atmosférica, ambas encontradas no ambiente de trabalho rural. Segundo o Ministério da Saúde do Brasil, a vulnerabilidade do sistema nervoso aos efeitos da exposição ocupacional e ambiental a uma gama de substâncias químicas, agentes físicos e fatores causais de adoecimento, decorrentes da organização do trabalho, tem ficado cada vez mais evidente, traduzindo-se em episódios isolados ou epidêmicos de doença nos trabalhadores. As manifestações neurológicas das intoxicações decorrentes da exposição ocupacional a metais pesados, a agrotóxicos ou a solventes orgânicos, e de outras doenças do sistema nervoso relacionadas às condições de trabalho, costumam receber o primeiro atendimento na rede básica de serviços de saúde 18 .

Outros estudos evidenciaram a relação entre a etiologia das neuropatias periféricas com a atividade ocupacional e se descobriu que o surgimento dessas lesões pode estar associado à exposição aos agentes neurotóxicos, como o chumbo, solventes orgânicos, pesticidas, a radiação ionizante e o frio ${ }^{19}$. Wechsler et. al. 20 avaliaram a associação da exposição a agentes tóxicos ambientais com a etiologia da doença de Parkinson. Os fatores de risco investigados neste estudo foram o tipo de ocupação, uso de água tratada, uso de pesticidas, exposição a metais, história clínica e tabagismo. Ao avaliar a exposição ocupacional a metais, as exposições ao alumínio e ao cobre tenderam a ser mais comuns entre os indivíduos expostos a essas substâncias em comparação ao grupo controle.

Outro estudo também associou esses fatores de risco, encontrando uma possível relação entre esses fatores com a frequência de doenças neurológicas nos indivíduos estudados 21 . Esses achados corroboram os resultados encontrados em neste estudo, considerando que o trabalhador rural é exposto a poluentes atmosféricos, além da utilização de pesticidas e agrotóxicos no campo de trabalho. 
Em relação ao número de dias afastados, na maioria dos atestados constou um dia de afastamento em todos os grupos de doenças analisadas. Entretanto, os afastamentos causados por doenças do aparelho geniturinário tiveram maior número de dias de afastamento. O Ministério da Saúde apontou que a incidência de doenças do aparelho geniturinário, como a cistite aguda, pode ocorrer em trabalhadores expostos a agentes que possuam ação irritante sobre a mucosa vesical como os encontrados em pesticidas fortemente utilizados no ambiente rural 18.

Além dos aspectos relacionados diretamente à saúde de trabalhadores, diversos fatores determinam o absenteísmo, como a cultura organizacional do trabalho, a ausência de estratégias de valorização do trabalhador, o estresse, a alta demanda energética exigida durante o trabalho, ambiente psicossocial desfavorável, a insatisfação com o trabalhador e a condição socioeconômica dos trabalhadores 22 .

Como limitações deste estudo, a falta de acesso a dados como idade, dados antropométricos, tempo de trabalho no setor canavieiro e exames admissionais não permitiram uma abordagem complexa em relação às características individuais e aos fatores de risco na população estudada. Apesar dessas limitações, os resultados reafirmam a multifatoriedade $\mathrm{e}$ a complexidade do absenteísmo por doenças ocupacionais no ambiente rural. Trata-se de um fenômeno a ser analisado sob a perspectiva do processo de trabalho, da cultura organizacional e de aspectos diretamente relacionados à saúde desses trabalhadores.

Desse modo, com base nos resultados obtidos, constata-se que, além das doenças do sistema osteomuscular e do tecido conjuntivo, as doenças do aparelho respiratório são as principais causas de afastamento de trabalhadores rurais no setor canavieiro. No entanto, as doenças do aparelho geniturinário são as que acarretam um maior número de dias de afastamento. Portanto, é fundamental a atuação de uma equipe multidisciplinar de profissionais da saúde que atentem para o desenvolvimento de atividades de ergonomia, equipamentos de proteção individual e orientações de saúde direcionadas especificamente às necessidades dessa população.

\section{Resumen}

El objetivo del estudio fue analizar la frecuencia de ausencias por enfermedades ocupacionales de trabajadores rurales en una zafra azucarera. Fueron analizados 1.230 certificados médicos de acuerdo con la 10a revisión de la Classificación Internacional de las Enfermedades (CID-10) de 400 trabajadores rurales. En los certificados se examinaron: diagnóstico médico, sexo, días de ausencia y período de ausencia. En los períodos descritos las enfermedades del aparato locomotor mostraron una mayor frecuencia en los certificados, seguidas de las enfermedades respiratorias $(p<0,05)$. Los certificados por enfermedades del aparato locomotor fueron más frecuentes al final que en al principio de la zafra $(p<0,05)$. En relación con el número de días de ausencias, un 75\% de los certificados tenían un día. Los certificados médicos de las enfermedades del sistema genitourinario tenían un mayor número de días de ausencias $(p<0,001)$. Por ello, es esencial trabajar con un equipo multidisciplinar de profesionales de la salud que se centren en el desarrollo de actividades de ergonomía, equipo de protección individual y orientación, dirigidos específicamente a las necesidades de esta población.

Trabajadores Rurales; Absentismo; Enfermedades

Profesionales 


\section{Colaboradores}

A. D. F. Ceccato, L. C. S. Carvalho Junior e R. C. Cuiss contribuíram com a redação do artigo e a revisão crítica relevante do conteúdo intelectual. M. Monteschi e N. G. Oliveira contribuíram com a redação do artigo. C. R. Padovani e E. M. C. Ramos contribuíram com a análise e a interpretação dos dados e aprovação final da versão a ser publicada. D. Ramos contribuiu com a concepção do projeto e com a aprovação final da versão a ser publicada.

\section{Agradecimentos}

Este trabalho foi financiado pela FAPESP, pelo Programa Institucional de Bolsas de Iniciação Científica (PIBIC/CNPq) e pelo Instituto Nacional de Análise Integrada do Risco Ambiental (INAIRA/CNPq).

\section{Referências}

1. União da Indústria de Cana-de-Açúcar. O maior projeto de capacitação e requalificação de cortadores de cana já realizado pelo setor sucroenergético. http://unica.com.br/Renovacao/ (acessado em 25/Set/2013).

2. Alves F. Por que morrem os cortadores de cana? Saúde Soc 2006; 15:90-8.

3. Ribeiro H, Ficarelli TRA. Queimadas nos canaviais e perspectivas dos cortadores de cana-de-açúcar em Macatuba, São Paulo. Saúde Soc 2010; 19:48-63.

4. Ribeiro H. Queimadas de cana-de-açúcar no Brasil: efeitos à saúde respiratória. Rev Saúde Pública 2008; 42:42-6.

5. Muller ML, Coetsee MF. Physiological demands and working efficiency of sugarcane cutters in harvesting burnt and unburnt cane. Int J Ind Ergon 2008; 38:314-20.

6. Teixeira MP, Freitas RMV. Acidentes do trabalho rural no interior paulista. São Paulo Perspect 2003; 17:81-90.

7. Rumin CR, Schmidt MLG. Influências das condições e organização de trabalho de uma indústria de transformação de cana-de-açúcar na ocorrência de acidentes de trabalho. Saúde Soc 2008; 17:56-67.
8. Silveira CA, Robazzi MLCC, Marziale MHP, Dalri MCB. Acidente de trabalho entre trabalhadores rurais e a agropecuária identificados através de registros hospitalares. Ciênc Cuid Saúde 2005; 4:1208.

9. Alessi NP, Navarro VL. Saúde e trabalho rural: o caso dos trabalhadores da cultura canavieira na região de Ribeirão Preto, São Paulo, Brasil. Cad Saúde Publica 1997; 13 Suppl 2:111-21.

10. Organização Mundial da Saúde. Classificação estatística internacional de doenças e problemas relacionados à saúde - CID-10. 8a Ed. São Paulo: Edusp; 2000.

11. Goodman LA. On simultaneous confidence intervals for multinomial proportions. Technometrics 1965; 7:247-54.

12. Filho VW. Perfil epidemiológico dos trabalhadores. Rev Bras Med Trab 2004; 2:103-17.

13. Simões RLM, Rocha AM, Souza C. Factors associated with absenteeism-illness in rural workers in a timber company. Rev Latinoam Enferm 2012; 20:718-26.

14. Davey MM, Cummings G, Newburn-Cook CV, Lo EA. Predictors of nurse absenteeism in hospitals: a systematic review. J Nurs Manag 2009; 17:312-30. 
15. Griep RH, Rotenberg L, Chor D, Toivanen S, Landisbergis P. Beyond simple approaches to studying the association between work characteristics and absenteeism: combining DCS and ERI models. Work Stress 2010; 24:179-95.

16. Goto DM, Lança M, Obuti CA, Barbosa CMG, Saldiva PHN, Zanetta DMT, et al. Effects of biomass burning on nasal mucociliary clearance and mucus properties after sugarcane harvesting. Environ Res 2011; 111:664-9.

17. Ferreira-Ceccato AD, Ramos EMC, CarvalhoJunior LCS, Xavier RF, Teixeira MFS, RaymundoPereira PA, et al. Short terms effects of air pollution from biomass burning in mucociliary clearance of brazilian sugarcane cutters. Respir Med 2011; 105:1766-8.

18. Dias EC, organizador. Doenças relacionadas ao trabalho: manual de procedimentos para os serviços de saúde. Brasília: Ministério da Saúde/Organização Pan-Americana da Saúde; 2001. (Série A. Normas e Manuais Técnicos, 114).
19. Souza A, Medeiros AR, Souza AC, Wink M, Siqueira IR, Ferreira MBC, et al. Avaliação do impacto da exposição a agrotóxicos sobre a saúde de população rural. Vale do Taquari (RS, Brasil). Ciênc Saúde Coletiva 2011; 16:3519-28.

20. Wechsler LS, Checkoway H, Franklin GM, Costa LG. A pilot study of occupational and environmental risk factors for Parkinson's disease. Neurotoxicology 1991; 12:387-93.

21. Gurgueira GP, Alexandre, NMC, Corrêa Filho HR. Prevalência de sintomas musculoesqueléticos em trabalhadoras de enfermagem. Rev Latinoam Enferm 2003; 11:608-13.

22. Minayo-Gomez C. Produção de conhecimento e intersetorialidade em prol das condições de vida e de saúde dos trabalhadores do setor sucroalcooleiro. Ciênc Saúde Coletiva 2011; 16:3361-8.

Recebido em 07/Fev/2013

Versão final reapresentada em 25/Set/2013 Aprovado em 18/Mar/2014 